\title{
Investigating the X-ray Emission from the Galactic TeV Gamma-ray Source MGRO J1908+06
}

\author{
Dirk Pandel* \\ Grand Valley State University, Allendale, MI 49401, USA \\ E-mail: pandeldagvsu.edu
}

\begin{abstract}
MGRO J1908+06 is a bright, extended TeV gamma-ray source located near the Galactic plane. The $\mathrm{TeV}$ emission has previously been attributed to the pulsar wind nebula of the radio-faint gamma-ray pulsar PSR J1907+0602 discovered with Fermi. However, studies of the TeV morphology with VERITAS have shown that MGRO J1908+06 is somewhat larger than other pulsar wind nebulae of similar age and that the $\mathrm{TeV}$ spectrum does not soften with distance from the pulsar as is observed for other pulsar wind nebulae. Although MGRO J1908+06 is very bright in gamma rays with a flux corresponding to $\sim 80 \%$ of the Crab Nebula flux at $20 \mathrm{TeV}$, no extended emission at other energies has so far been detected. We report on our analysis of X-ray data obtained with XMM-Newton of the region near MGRO J1908+06. We searched the data for point-like sources and detected several hard-spectrum X-ray sources that could be associated with the TeV emission, including the gamma-ray pulsar PSR J1907+0602. We also performed an extended source analysis to search for diffuse emission from MGRO J1908+06 but found no evidence of diffuse $\mathrm{X}$-ray emission coincident with the TeV source. We place an upper limit of $8.7 \times 10^{33} \mathrm{erg} \mathrm{s}^{-1}$ on the X-ray luminosity of MGRO J1908+06 in the 1-10 keV energy range. The corresponding limit on the ratio of gamma-ray to X-ray luminosity is consistent with the ratios found for other pulsar wind nebulae of similar age.
\end{abstract}

The 34th International Cosmic Ray Conference

30 July - 6 August, 2015

The Hague, The Netherlands

${ }^{*}$ Speaker. 


\section{Introduction}

In a survey of the Galactic plane at a median energy of $20 \mathrm{TeV}$, the Milagro collaboration discovered the bright TeV gamma-ray source MGRO J1908+06 with a flux of $\sim 80 \%$ of the Crab Nebula flux at these energies [1]. The source was subsequently detected with H.E.S.S. above $300 \mathrm{GeV}[2,3]$ and with VERITAS [4]. The H.E.S.S. observations showed that MGRO J1908+06 (HESS J1908+063) is an extended source, and a fit of a two-dimensional Gaussian function to the excess map yielded an intrinsic size of the $\mathrm{TeV}$ source of $\sigma=0.34^{\circ}$ and a centroid position of R.A. $=19^{\mathrm{h}} 07^{\mathrm{m}} 54^{\mathrm{s}} .3$ and Decl. $=+06^{\circ} 16^{\prime} 07^{\prime \prime}\left(l=40^{\circ} 23^{\prime} 9^{\prime \prime}\right.$ and $\left.b=-0^{\circ} 47^{\prime} 10^{\prime \prime}\right)$ [3]. Although the $\mathrm{TeV}$ source is comparatively bright with a flux of $17 \%$ of that of the Crab Nebula above $1 \mathrm{TeV}$, no extended emission associated with MGRO J1908+06 has so far been detected at other energies. In particular, no extended $\mathrm{GeV}$ emission was found with the Fermi Large Area Telescope (LAT), which suggests that the spectrum of MGRO J1908+06 has a low-energy turnover between 20 and $300 \mathrm{GeV}$ [5].

MGRO J1908+06 may be associated with the nearby shell-type supernova remnant (SNR) G40.5-0.5. However, the TeV emission extends well beyond the boundary of the radio SNR, which would require either an additional source of gamma rays or the presence of dense molecular matter interacting with ultra-relativistic particles in the vicinity of the SNR [3]. A point-like GeV gamma-ray source, OFGL J1907.5+0602, was discovered with the Fermi LAT near the position of MGRO J1908+06 [6]. The source was later identified as a radio-quiet gamma-ray pulsar, PSR J1907+0602, with a pulse period of $106.6 \mathrm{ms,}$, a spin-down power of $\sim 2.8 \times 10^{36} \mathrm{erg} \mathrm{s}^{-1}$, and a characteristic age of $19.5 \mathrm{kyr}$ [7, 8]. Using a time differencing technique, a precise position of the pulsar of R.A. $=19^{\mathrm{h}} 07^{\mathrm{m}} 54^{\mathrm{s}} .7(2)$ and Decl. $=+06^{\circ} 02^{\prime} 16(2)^{\prime \prime}$ was derived from the Fermi data. Multi-wavelength observations of PSR J1907+0602 revealed a faint X-ray counterpart as well as faint, pulsed radio emission [8]. The dispersion measure obtained from the radio observations indicates a distance of $3.2 \pm 0.6 \mathrm{kpc}$ to the pulsar. The pulsar's position is offset by $\sim 0.23^{\circ}$ from the center of HESS J1908+063 but firmly within the extent of the TeV source. The close proximity of the two sources suggests that the $\mathrm{TeV}$ emission is the pulsar wind nebula (PWN) of PSR J1907+0602. The pulsar may also be associated with the SNR G40.5-0.5 [8]. The estimated age (20-40 kyr [9]) and distance (3.6 kpc [10]) of the SNR are consistent with those of the pulsar. However, if the pulsar originated at the center of the SNR, its characteristic age would imply a transverse velocity of $\sim 1400 \mathrm{~km} \mathrm{~s}^{-1}$. This is several times higher than typical pulsar velocities, although similar velocities have been observed in a few cases [11]. At such a high pulsar velocity, a bow shock and pulsar tail will likely be present at X-ray or radio energies. Evidence of a bow shock consistent with the pulsar moving away from the SNR was found in X-ray images obtained with XMM-Newton [12].

Deep TeV observations of MGRO J1908+06 were carried out with VERITAS to investigate the morphology of the source [11]. The TeV emission was found to extend from the region near PSR J1907+0602 to the boundary of the SNR G40.5-0.5. However, in contrast to other PWNe of similar age, the $\mathrm{TeV}$ spectrum does not soften with distance from the pulsar. This is inconsistent with the TeV emission near the SNR being solely due to the PWN of PSR J1907+0602. If the pulsar was born at the center of the SNR, the leptons injected into the PWN closer to the pulsar's birthplace would have cooled significantly via synchrotron radiation and inverse Compton scattering, leading 
to a softer TeV spectrum near the SNR [11]. It has therefore been suggested that the $\mathrm{TeV}$ emission near the SNR is caused by the interaction of the pulsar wind with nearby molecular clouds or the SNR shock. It is also possible that another PWN, associated with an as yet undetected pulsar along the line of sight, contributes to the $\mathrm{TeV}$ emission.

We present an analysis of X-ray data obtained with XMM-Newton [13] of the region near MGRO J1908+06. The XMM-Newton observations and our analysis of the X-ray data are described in $\S 2$. In $\S 3$ we discuss our search for point-like X-ray sources that may be associated with the TeV source, and in $\$ 4$ we investigate the diffuse X-ray emission from MGRO J1908+06. Our findings are discussed in $\S 5$.

\section{Observations and Analysis}

MGRO J1908+06 was observed with XMM-Newton during four observations with exposure times ranging from 7 to $18 \mathrm{ks}$ (Observation IDs 0553640101, 0553640201, 0553640701, and 0553640801). Because of the large extent of the TeV source, the observations were arranged in a 2-by-2 mosaic covering a field $0.75^{\circ}$ across with the X-ray cameras. We analyzed the data using the XMM-Newton Science Analysis Software (SAS) and included in our analysis data from the two EPIC MOS cameras [14] and the EPIC PN camera [15].

To search for point-like X-ray sources, we used the SAS task emosaicproc with the combined data from the three cameras and four observations in the $0.4-10 \mathrm{keV}$ energy range. We detected 22 sources with a likelihood $D E T \_M L>15$ which corresponds to a probability of $<3 \times 10^{-7}$ for the observed source counts to be caused by a background fluctuation. For each of the detected sources, we extracted a background-subtracted spectrum using a $20^{\prime \prime}$ circular aperture and determined the hardness ratio $H R=\left(F_{2-10 \mathrm{keV}}-F_{0.5-2 \mathrm{keV}}\right) / F_{0.5-10 \mathrm{keV}}$ of the observed fluxes in the $0.5-2 \mathrm{keV}$ and $2-10 \mathrm{keV}$ energy ranges.

To investigate the diffuse X-ray emission from MGRO J1908+06, we analyzed the data using the Extended Source Analysis Software ${ }^{1}$ (ESAS). We excluded from our analysis time intervals with strong contamination by soft proton flares and EPIC MOS CCDs that were operating in an anomalous state. Using the ESAS, we modeled the particle background for each observation and camera and created background-subtracted images and spectra while taking into account the varying effective exposure times across the field of view. An image of the combined X-ray data from the three cameras and four observations is shown in Figure 1. To obtain spectra of the diffuse X-ray emission for selected regions, we excluded all detected point sources and combined the remaining data from all observations for each camera. Because of the large extent of MGRO J1908+06, it was not possible to estimate the residual soft proton background from a region away from the $\mathrm{TeV}$ source. We therefore did not subtract this background directly, but instead included it as a separate model component when fitting the spectrum. Our spectral analysis was restricted to the energy range $0.4-10 \mathrm{keV}$ for the EPIC MOS cameras and $0.4-12 \mathrm{keV}$ for the EPIC PN camera. To avoid strong emission lines in the instrumental background that are difficult to model, we also excluded the 1.2-2.0 keV energy range for all cameras and the 7.2-9.2 keV energy range for the EPIC PN camera. The spectral analysis was performed using XSPEC [16].

\footnotetext{
${ }^{1} \mathrm{ftp} / / /$ legacy.gsfc.nasa.gov/xmm/software/xmm-esas/xmm-esas.pdf
} 


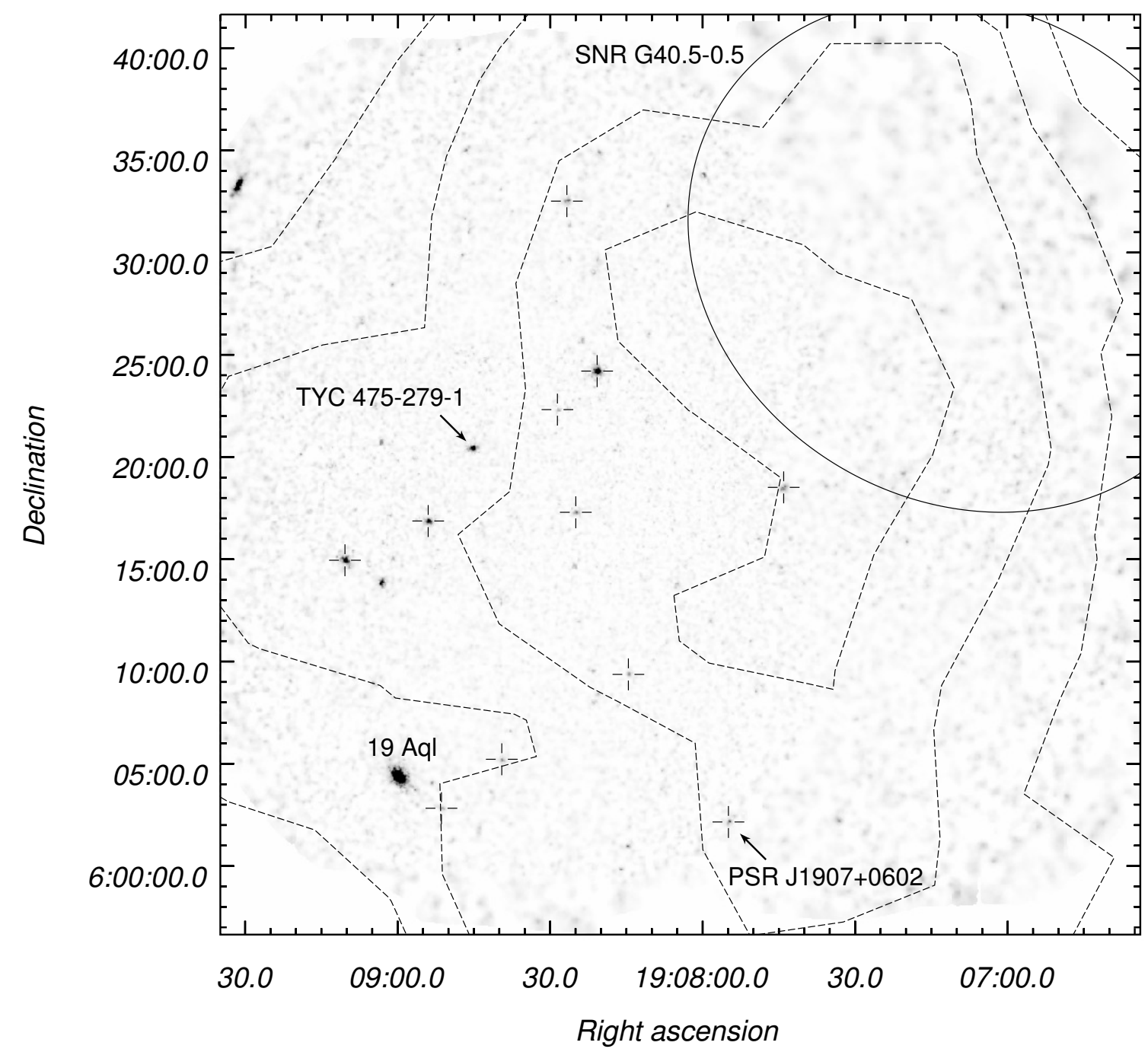

Figure 1: X-ray image of the region near MGRO J1908+06 in the $0.4-7.2 \mathrm{keV}$ energy range. The image is $0.75^{\circ}$ across and shows the combined data from the three $\mathrm{X}$-ray cameras and four observations. The image has been exposure corrected, background subtracted (except for the residual soft proton background and some instrumental lines between 1.2 and $2.0 \mathrm{keV}$ ), and adaptively smoothed. The crosses indicate the positions of the detected X-ray sources with a hard spectrum (hardness ratio $H R>0$ ). The dashed contours show a significance map $(7 \sigma, 6 \sigma, 5 \sigma$, and $4 \sigma$ levels $)$ of the $\mathrm{TeV}$ gamma-ray emission detected with H.E.S.S. (from [2]). The ellipse outlines the extent of the shell-type SNR G40.5-0.5. Note that the effective exposure time varies significantly across the image, leading to an uneven appearance of the diffuse emission after the adaptive smoothing is applied.

\section{X-ray Point Sources}

Of the 22 point-like X-ray sources we detected, three are previously known sources observed at other wavelengths: two foreground stars with a soft X-ray spectrum, 19 Aql and TYC 475-279-1, and the gamma-ray pulsar PSR J1907+0602. The X-ray spectrum we found for PSR J1907+0602 is consistent with that obtained from Chandra observations [8]. Sources with a hard X-ray spectrum 
MGRO J1908+06 Region

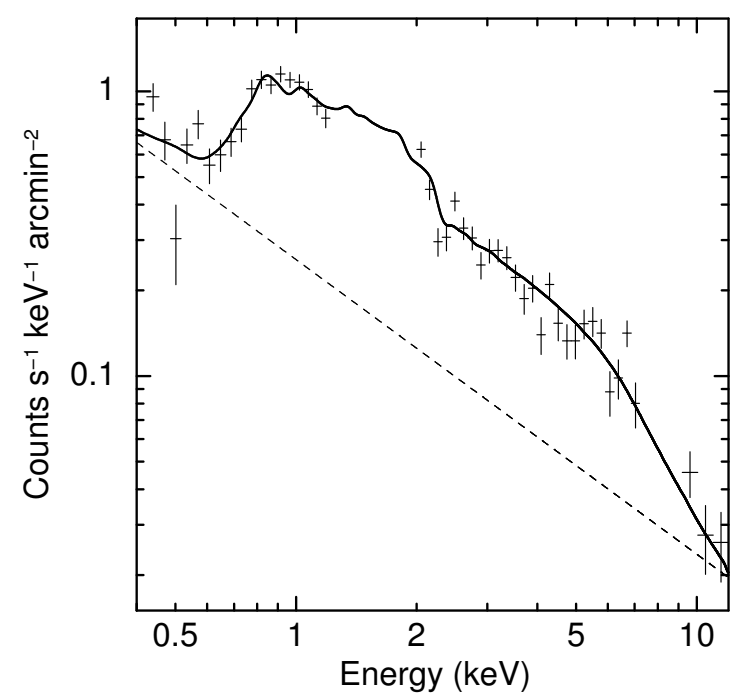

Background Region

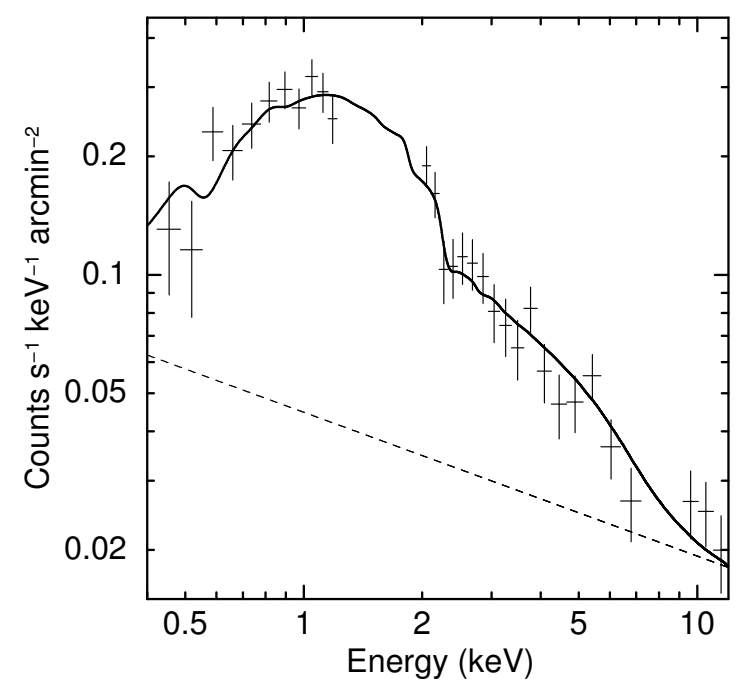

Figure 2: Spectrum of the diffuse X-ray emission from a $45^{\prime}$ circular region around MGRO J1908+06 and from a background region (see §4). The data points show the particle background subtracted count rates measured with the EPIC PN camera normalized to an area of one square arcminute. The solid curve is a best fit with an absorbed mekal plus power law model (left) or an absorbed power law model (right). The dashed line shows an estimate of the residual soft proton background obtained from the fit.

(hardness ratio $H R>0$ ) are marked with crosses in Figure 1. Note that the effective exposure time on the right side of the figure is $\sim 2.5$ times lower than on the left side, which may explain the larger number of detected sources on the left.

A possible explanation for the TeV emission away from PSR J1907+0602 (near the left edge of SNR G40.5-0.5 in Figure 1) is the presence of a second PWN associated with an as yet undetected pulsar along the line of sight [11]. We detected several sources with a hard X-ray spectrum in this region which are potential candidates for such a pulsar. However, further multi-wavelength observations are necessary to determine if any of these sources are pulsars or compact X-ray PWNe.

\section{Diffuse X-ray Emission}

Although some diffuse X-ray emission not associated with the point-like sources is visible in Figure 1, it appears to be uniform and does not show any excess correlated with the $\mathrm{TeV}$ emission (dashed contours). To investigate the spectrum of the diffuse X-ray emission near MGRO J1908+06 we excluded the detected point sources and selected all remaining photons from a circular region with a radius of $45^{\prime}$ centered on R.A. $=19^{\mathrm{h}} 07^{\mathrm{m}} 22^{\mathrm{s}}$ and Decl. $=+06^{\circ} 13^{\prime} 12^{\prime \prime}$, the centroid position of the TeV emission determined with VERITAS [11]. To obtain a spectrum of the diffuse background emission, we selected all photons outside the $45^{\prime}$ circular region. Figure 2 shows the count rate spectra from the EPIC PN camera for the MGRO J1908+06 region and the background region normalized to an area of one square arcminute.

We fitted the spectrum of the MGRO J1908+06 region with a model for a hot diffuse gas (mekal) plus a power-law model and included photoelectric absorption by the interstellar medium. 


\begin{tabular}{lcc}
\hline & MGRO J1908+06 Region & Background Region \\
\hline Flux in $10^{-14} \mathrm{erg} \mathrm{cm}^{-1} \mathrm{~s}^{-1} \mathrm{arcmin}^{-2}$ & & \\
$\quad$ Absorbed & $1.05 \pm 0.11$ & $1.12 \pm 0.17$ \\
$\quad$ Unabsorbed & $1.18 \pm 0.10$ & $1.18 \pm 0.16$ \\
Flux in $10^{-6}$ photons cm & \\
$\quad$ Absorbed & & \\
Unabsorbed & $1.82 \pm 0.12$ & $2.02 \pm 0.22$ \\
\hline
\end{tabular}

Table 1: Absorbed and unabsorbed X-ray flux per square arcminute in the 1-10 keV energy range from a $45^{\prime}$ circular region around MGRO J1908+06 and from a background region (see §4). Uncertainties are given at the $68 \%$ confidence level.

The spectrum of the background region was fitted with an absorbed power law model. The best fits with these models are shown in Figure 2 as solid curves. Although we were able to estimate and subtract most of the instrumental background, the spectra still contain a residual contribution from the soft proton background. We modeled this background separately for each camera as a power law that is not folded through the instrumental response and included it as an additional model component when fitting the spectrum (dashed lines in Figure 2).

From the spectral fits we estimated the average energy flux and photon flux per square arcminute for the two regions in the $1-10 \mathrm{keV}$ energy range. Table 1 shows the absorbed flux observed with XMM-Newton as well as the unabsorbed flux obtained after removing the effects of interstellar absorption. The X-ray flux in the MGRO J1908+06 region is consistent within uncertainties with the flux in the background region. We therefore find no evidence of any X-ray emission coincident with the TeV emission from MGRO J1908+06. From the flux measurements we derive an upper limit of $7.1 \times 10^{-12} \mathrm{erg} \mathrm{cm}^{-1} \mathrm{~s}^{-1}$ or $1.4 \times 10^{-3}$ photons $\mathrm{cm}^{-1} \mathrm{~s}^{-1}$ (95\% confidence level) on the $1-10 \mathrm{keV}$ X-ray flux from MGRO J1908+06.

\section{Discussion}

According to current models, the emission from PWNe is produced by a population of leptons accelerated to very high energies at the shocks formed by the pulsar wind interacting with the ambient medium. The spectrum of the emission has two broad components, one at X-ray to radio energies generally understood as synchrotron radiation from the highly energetic leptons, and one at gamma-ray energies caused by Inverse Compton (IC) scattering of ambient low-energy photons. Because of the spin down of the pulsar and the cooling of the leptons, both spectral components decrease with the age of the PWN. However, the decaying magnetic field causes the synchrotron component to decrease more rapidly than the IC component [17]. In agreement with this model, the data compiled by [18] on known PWNe show a positive correlation between the gamma-ray to $\mathrm{X}$-ray luminosity ratio $L_{\gamma} / L_{X}$ and the pulsar age.

Our upper limit on the 1-10 keV X-ray flux from MGRO J1908+06 corresponds to a luminosity of $L_{X} \leq 8.7 \times 10^{33} \mathrm{erg} \mathrm{s}^{-1}$ for a distance of $3.2 \mathrm{kpc}$. According to Fig. 2 in [18], this upper limit is $1-2$ orders of magnitude higher than the X-ray luminosity of most PWNe containing a pulsar with a similar spin-down power as PSR J1907+0602 $\left(\sim 2.8 \times 10^{36} \mathrm{erg} \mathrm{s}^{-1}\right)$. The gamma-ray luminosity of MGRO J1908+06 is $L_{\gamma}=3 \times 10^{34} \mathrm{erg} \mathrm{s}^{-1}$ in the $1-10 \mathrm{TeV}$ energy range [11]. This 


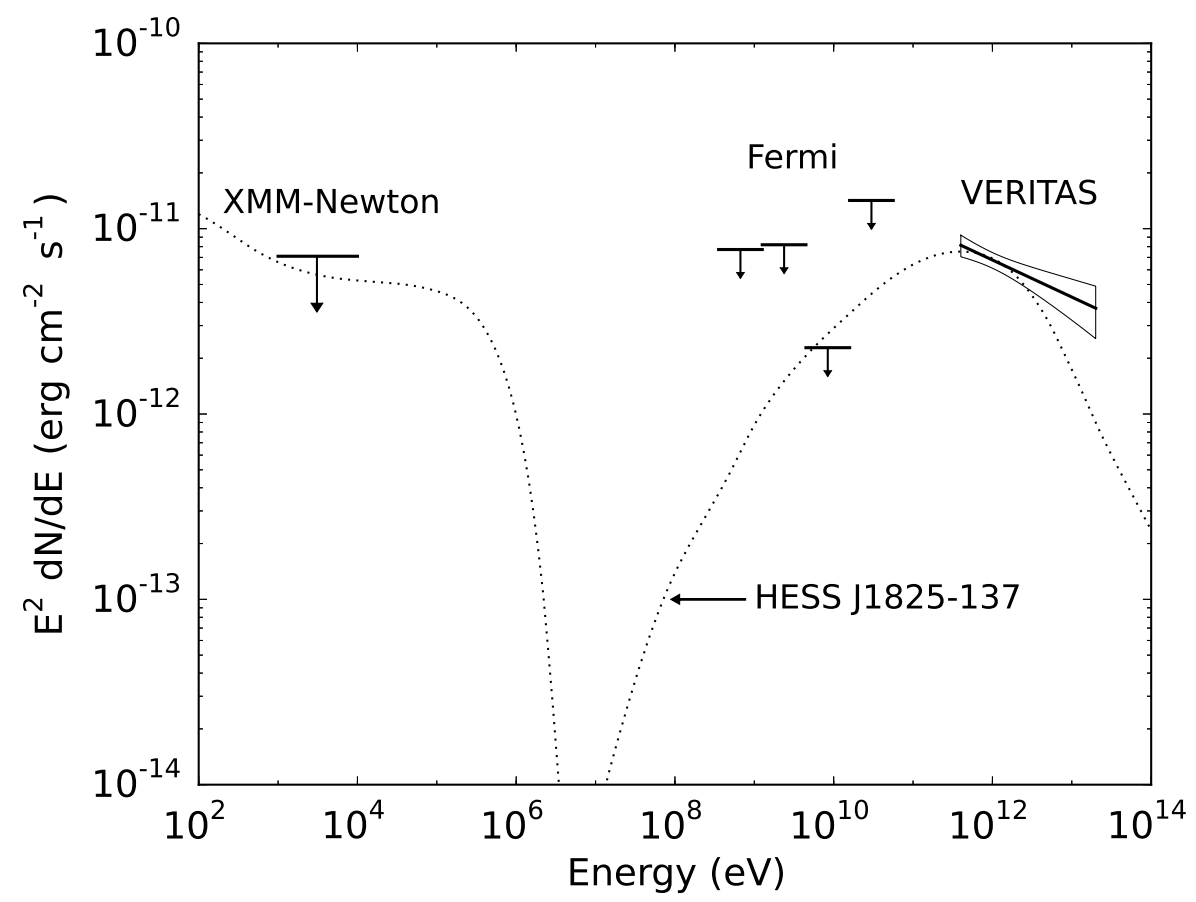

Figure 3: TeV spectrum of MGRO J1908+06 obtained with VERITAS [11] shown together with the Fermi upper limits on the off-pulse $\mathrm{GeV}$ emission from the pulsar region [8] and our upper limit on the X-ray flux. For comparison, the dotted curve shows a modeled spectral energy distribution of the PWN HESS J1825-137 scaled to match the TeV flux of MGRO J1908+06 (from [19]).

implies a lower limit on the gamma-ray to X-ray luminosity ratio of $L_{\gamma} / L_{X} \gtrsim 3$. For other PWNe with a similar age as MGRO J1908+06 ( 19.5 kyr), this ratio is typically in the 1-500 range (Fig. 4 in [18]). We therefore conclude that the non-detection of X-ray emission from MGRO J1908+06 is consistent with the X-ray luminosities found for other PWNe with a similar pulsar age and spindown power as PSR J1907+0602.

Time-dependent models that take into account the injection and cooling of leptons in PWNe have been used to investigate the evolution of the spectral energy distribution from radio to $\mathrm{TeV}$ energies [17, 19]. Such models could be used to predict the X-ray luminosity of MGRO J1908+06 from the observed $\mathrm{TeV}$ spectrum or to constrain certain PWN parameters such as the magnetic field strength. Figure 3 shows the TeV spectrum of MGRO J1908+06 together with our upper limit on the X-ray flux and the Fermi upper limits on the off-pulse GeV emission [8]. For comparison, the figure also shows a modeled spectral energy distribution of the PWN HESS J1825-137 (from [19]) which has a similar age and pulsar spin-down power as MGRO J1908+06.

\section{Acknowledgments}

This work is based on observations obtained with XMM-Newton, an ESA science mission with instruments and contributions directly funded by ESA member states and the USA (NASA). This material is based upon work supported by NASA Guest Investigator Grant NAG57816 and by the National Science Foundation under Grant No. 1068152. 


\section{References}

[1] A. A. Abdo, et al., TeV Gamma-Ray Sources from a Survey of the Galactic Plane with Milagro, ApJ 664, L91 (2007).

[2] E. de Oña Wilhelmi, A. Djannati-Atai, M. Renaud, The unidentified source HESS J1908+063 / MGRO J1908+06, in HIGH ENERGY GAMMA-RAY ASTRONOMY: 4th International Meeting on High Energy Gamma-Ray Astronomy, AIP Conf. Proc. 1085, 273 (2008).

[3] F. Aharonian, et al., Detection of very high energy radiation from HESS J1908+063 confirms the Milagro unidentified source MGRO J1908+06, A\&A 499, 723 (2009).

[4] J. E. Ward, VERITAS Observations of MGRO J1908+O6 / HESS J1908+063, in HIGH ENERGY GAMMA-RAY ASTRONOMY: 4th International Meeting on High Energy Gamma-Ray Astronomy, AIP Conf. Proc. 1085, 301 (2008).

[5] M. Ackermann, et al., Fermi-LAT Search for Pulsar Wind Nebulae Around Gamma-ray Pulsars, ApJ 726, 35 (2011).

[6] A. A. Abdo, et al., Fermi/Large Area Telescope Bright Gamma-Ray Source List, ApJS 183, 46 (2009).

[7] A. A. Abdo, et al., Detection of 16 Gamma-Ray Pulsars Through Blind Frequency Searches Using the Fermi LAT, Science 325, 840 (2009).

[8] A. A. Abdo, et al., PSR J1907+0602: A Radio-Faint Gamma-Ray Pulsar Powering a Bright TeV Pulsar Wind Nebula, ApJ 711, 64 (2010).

[9] A. J. B. Downes, C. J. Salter, T. Pauls, G40.5-0.5 - A previously unrecognised supernova remnant in Aquila, A\&A 92, 47 (1980).

[10] J. Yang, et al., Molecular Gas Distribution around the Supernova Remnant G40.5-0.5, ChJAA 6, 210 (2006).

[11] E. Aliu, et al., Investigating the TeV Morphology of MGRO J1908+06 with VERITAS, ApJ 787, 166 (2014).

[12] D. Pandel, R. Scott, Multi-wavelength studies of the gamma-ray pulsar PSR J1907+0602, in HIGH ENERGY GAMMA-RAY ASTRONOMY: 5th International Meeting on High Energy Gamma-Ray Astronomy, AIP Conf. Proc. 1505, 329 (2012).

[13] F. Jansen, et al., XMM-Newton observatory. I. The spacecraft and operations, A\&A 365, L1 (2001).

[14] M. J. L. Turner, et al., The European Photon Imaging Camera on XMM-Newton: The MOS cameras, $A \& A$ 365, L27 (2001).

[15] L. Strüder, et al., The European Photon Imaging Camera on XMM-Newton: The pn-CCD camera, $A \& A$ 365, L18 (2001).

[16] K. A. Arnaud, XSPEC: The First Ten Years, in Astronomical Data Analysis Software and Systems V, ASP Conf. Series 101, 17 (1996).

[17] S. J. Tanaka, F. Takahara, A Model of the Spectral Evolution of Pulsar Wind Nebulae, ApJ 715, 1248 (2010).

[18] O. Kargaltsev, B. Rangelov, G. G. Pavlov, Gamma-ray and X-ray Properties of Pulsar Wind Nebulae and Unidentified Galactic TeV Sources, eprint arXiv:1305.2552 (2013).

[19] M. Mayer, J. Brucker, M. Holler, I. Jung, K. Valerius, C. Stegmann, Predicting the X-ray flux of evolved pulsar wind nebulae based on VHE gamma-ray observations, eprint arXiv:1202.1455 (2012). 